\title{
COMPLEX SUBMANIFOLDS IN REAL HYPERSURFACES
}

\author{
Chong-Kyu Han and Giuseppe Tomassini
}

\begin{abstract}
Let $M$ be a $C^{\infty}$ real hypersurface in $\mathbb{C}^{n+1}, n \geq 1$, locally given as the zero locus of a $C^{\infty}$ real valued function $r$ that is defined on a neighborhood of the reference point $P \in M$. For each $k=1, \ldots, n$ we present a necessary and sufficient condition for there to exist a complex manifold of dimension $k$ through $P$ that is contained in $M$, assuming the Levi form has rank $n-k$ at $P$. The problem is to find an integral manifold of the real 1 -form $i \partial r$ on $M$ whose tangent bundle is invariant under the complex structure tensor $J$. We present generalized versions of the Frobenius theorem and make use of them to prove the existence of complex submanifolds.
\end{abstract}

\section{$\S 1$. Introduction}

Let $M$ be a real hypersurface in $\mathbb{C}^{n+1}, n \geq 1$, of class $C^{k}, k \geq 2$. Locally at a point $P \in M, M$ divides $\mathbb{C}^{n+1}$ in two connected components $U^{ \pm}$. Let $\mathcal{O}_{P}$ denote the ring of germs of holomorphic functions at $P$ and $\mathcal{O}\left(U^{ \pm}\right)$the spaces of holomorphic functions in $U^{ \pm}$. We say that $M$ has the local extension property at $P$ if there exists a fundamental system $\mathcal{U}=\left\{U_{\nu}\right\}, \nu=1,2, \ldots$ of open neighbourhoods of $P$ in $\mathbb{C}^{n+1}$ such that

i) $U_{\nu} \backslash M$ has two connected components $U_{\nu}^{+}, U_{\nu}^{-}$;

ii) for one of $U_{\nu}^{+}, U_{\nu}^{-}$holomorphic functions extend holomorphically through $P$.

If the Levi-form of $M$ has a non-zero eigenvalue at $P$ and if $M$ is of class $C^{3}$, then $M$ has the extension property at $P$ by the extension theorem due to H. Lewy ([13], [1]). If $M$ contains a germ at $P$ of a complex hypersurface $\{f=0\}$ it has not the extension property at $P$ for $1 / f$ is a holomorphic function in $U_{\nu}^{+}$(also in $U_{\nu}^{-}$) that does not extend through $P$. By a theorem of Trépreau [14] the converse is true, that is, $M$ does not have the extension property if and

Received January 5, 2009.

2000 Mathematics Subject Classification. 32D15, 58A10, 58A17.

Key words and phrases. extension of holomorphic functions, real hypersurfaces in complex manifolds, complex submanifolds, Levi-form, Pfaffian system, generalized Frobenius theorem.

First author was partially supported by the Korea Research Foundation Grant 2005-070C00007.

Second author was partially supported by the MURST project "Geometric Properties of Real and Complex Manifolds" and GNSAGA of INDAM. 
only if there exists a complex hypersurface in $M$ through $P$. As for the complex manifolds of higher codimensions, although not much has been clarified yet, it seems to the authors that the existence of complex submanifolds in $M$ of higher codimensions is the obstruction to the extendibility of the $\bar{\partial}$-cohomology classes and also various analytic objects that are defined in one of $U^{ \pm}$.

In this paper, we find necessary and sufficient conditions for $M$ to contain a germ at $P$ of a complex submanifold of given a dimension. Suppose that $r$ is a local defining function of $M$, that is, $r$ is a $C^{\infty}$ non-degenerate $(d r \neq 0)$ real-valued function defined on a neighborhood $U$ of $P$ so that $M$ is locally the zero locus of $r$. Then

$$
\theta:=\sqrt{-1} \partial r
$$

is a real 1-form which defines a subbundle $H(M)$ of the maximal complex subspaces of $T M$ and

$$
d \theta=\sqrt{-1} \bar{\partial} r
$$

is the Levi-form, as we shall discuss in $\S 3$. Then a submanifold $N$ is a complex submanifold of $M$ if and only if $N$ is an integral manifold of $\theta$ and the tangent bundle $T N$ is invariant under the complex structure tensor $J$ of $\mathbb{C}^{n+1}$ (Proposition 3.5). Given a 1 -form $\theta$, the classical Pfaff problem is to determine its integral manifolds of maximal dimension.

Definition 1. Let $\theta$ be a 1 -form defined on a neighborhood $U$ of a manifold $M$. The integer $k$ defined by

$$
(d \theta)^{k} \wedge \theta \neq 0, \quad(d \theta)^{k+1} \wedge \theta=0
$$

is called the rank of $\theta$.

Finding the integral manifolds is clarified by the normal form, given by the following theorem whose proof is found in [2, Chapter 2]:

Theorem 2 (The Pfaff problem). Let $\theta$ be a 1-form of rank $k$ defined on an open neighborhood $U$ of a manifold $M$ of dimension $m$. Then there exists a coordinate system $y^{1}, \ldots, y^{m}$, possibly in a smaller neighborhood, such that

$$
\theta=d y^{1}+y^{2} d y^{3}+\cdots+y^{2 k} d y^{2 k+1} \text {. }
$$

Then the submanifolds given by $y^{2 j+1}=$ constant, $j=0,1, \ldots, k$, are integral manifolds of (1). Thus we have:

Corollary 3. If $\theta$ has constant rank $k$, then there exists $a(k+1)$-parameter family of integral manifolds of dimension $m-(k+1)$. Thus $M$ is locally foliated by integral manifolds of dimension $m-(k+1)$.

Notice that the case $k=0$ is the Frobenius integrability. In our problem this is the case that $M$ is Levi-flat and foliated by complex hypersurfaces. However, if $\theta$ has constant rank $k \geq 1$, this normal form does not seem to be immediately useful for the following reasons: we require integral manifolds to be complex manifolds and also we want to know not only the foliation by 
complex submanifolds but also the existence of a single complex submanifold. In order to find a complex submanifold of complex dimension $n-k$ under the condition that the Levi form has rank $k$, we make use of generalized versions of the Frobenius theorem that we present in $\S 2$. For the sake of computational convenience we use the ambient coordinates $(z, w):=\left(z_{1}, \ldots, z_{n}, w\right)$ of $\mathbb{C}^{n+1}$ rather than working on the real hypersurface $M$. Our argument in this paper is purely local: we work on a small neighborhood of a reference point and often we need to shrink the neighborhood to a smaller open set as our argument proceeds.

The authors express thanks to Alberto Saracco and Guy Roos for their interest and for many valuable discussions. Recently, by using the methods as in [5], N. Q. Dieu obtained results [6] on the existence of complex hypersurfaces in real subvarieties defined by real homogeneous polynomials.

\section{$\S$ 2. Integral manifolds for Pfaffian systems}

In this section we present a theory with an algorithm of finding integral manifolds for the general case of Pfaffian system of several 1-forms. We adopt the definitions and notations from the references [2] and [8]. Let $M$ be a $C^{\infty}$ real manifold of dimension $m$. A Pfaffian system is a system of $C^{\infty}$ real 1-forms that are linearly independent at every point of $M$ :

$$
\theta:=\left(\theta^{1}, \ldots, \theta^{s}\right) \text {. }
$$

Let $p:=m-s$. A $C^{\infty}$ real submanifold $N$ of dimension $n, n \leq p$, is called an integral manifold of the Pfaffian system $\theta$ if

$$
i^{*} \theta^{\alpha}=0, \quad \forall \alpha=1, \ldots, s,
$$

where $i: N \hookrightarrow M$ is the inclusion map. Let

$$
\Omega^{*}:=\bigoplus_{k=0}^{m} \Omega^{k}
$$

be the exterior algebra of $C^{\infty}$ differential forms of $M$, where $\Omega^{k}$ is the set of smooth $k$-forms and $\Omega^{0}:=C^{\infty}(M)$ is the ring of smooth complex-valued functions on $M$. Each $\Omega^{k}$ is a module over $C^{\infty}(M)$. A subalgebra $\mathcal{I}$ is called an algebraic ideal if $\mathcal{I} \wedge \Omega^{*} \subset \mathcal{I}$ and if the following additional condition is satisfied: if $\phi=\sum_{k=0}^{m} \phi_{k} \in \mathcal{I}$, where $\phi_{k} \in \Omega^{k}$, each component $\phi_{k} \in \mathcal{I}$ (homogeneity condition).

For $\Psi_{1}, \Psi_{2} \in \Omega^{*}$ and an algebraic ideal $\mathcal{I}$ we write

$$
\Psi_{1} \equiv \Psi_{2}, \quad \bmod \mathcal{I}
$$

if and only if $\Psi_{1}-\Psi_{2} \in \mathcal{I}$. Real-valued functions $\rho_{1}, \ldots, \rho_{d}$ shall be said to be non-degenerate if

$$
d \rho_{1} \wedge \cdots \wedge \rho_{d} \neq 0, \quad \bmod \left(\rho_{1}, \ldots, \rho_{d}\right),
$$


where $\left(\rho_{1}, \ldots, \rho_{d}\right)$ is the algebraic ideal generated by $\left(\rho_{1}, \ldots, \rho_{d}\right)$. The common zero locus of non-degenerate real functions is a smooth submanifold of $M$.

Now let $\mathcal{I}$ be the algebraic ideal generated by $\theta$, which is the set of all finite sums of the form $\sum \theta^{\alpha} \wedge \phi, \phi \in \Omega^{k}$ for some $k$. An algebraic ideal $\mathcal{I}$ is said to be closed if

$$
d \mathcal{I} \subset \mathcal{I}
$$

Then the following are equivalent:

a) $\mathcal{I}$ is closed.

b) For each $\alpha=1, \ldots, s$,

$$
d \theta^{\alpha}=0, \quad \bmod \left(\theta^{1}, \ldots, \theta^{s}\right) .
$$

A Pfaffian system $\theta=\left(\theta^{1}, \ldots, \theta^{s}\right)$ is said to be integrable (or involutive) if it satisfies (2.1). Then the Frobenius theorem states as follows:

Theorem 2.1. Let $M$ be a smooth manifold of dimension $m$ and let $\theta=$ $\left(\theta^{1}, \ldots, \theta^{s}\right)$ be a system of smooth real 1 -forms that are linearly independent at every point of $M$. If $\theta$ satisfies the integrability condition (2.1), then for any point $x \in M$ there exists a unique integral manifold $N$ of dimension $p:=$ $m-s$ through $x$ on a neighborhood of $x$. Therefore, $M$ is locally foliated by s-parameter family of integral manifolds.

Now we prove the following:

Theorem 2.2. Let $M^{m}$ be a smooth manifold and let $\theta:=\left(\theta^{1}, \ldots, \theta^{s}\right)$ be a system of smooth real 1 -forms that are linearly independent at every point of $M$. Let $n$ be an integer such that $2 \leq n \leq p:=m-s$. Suppose that $i: N^{n} \hookrightarrow M^{m}$ is a submanifold of dimension $n$, defined by $\rho_{1}=\cdots=\rho_{m-n}=0$, where $\rho:=$ $\left(\rho_{1}, \ldots, \rho_{m-n}\right)$ are smooth nondegenerate real-valued functions of $M$. Then the following are equivalent:

(i) $i^{*} \theta^{\alpha}=0, \quad \alpha=1, \ldots, s$.

(ii) $\forall \alpha=1, \ldots, s, \theta^{\alpha} \equiv 0, \bmod \left(\rho_{1}, \ldots, \rho_{m-n}, d \rho_{1}, \ldots, d \rho_{m-n}\right)$.

It is easy to show the following:

Lemma 2.3. Let $(t, x)$, where $t=\left(t_{1}, \ldots, t_{d}\right), x=\left(x_{1}, \ldots, x_{n}\right)$, be the standard coordinates of $\mathbb{R}^{d+n}$. Suppose that $f$ is a $C^{\infty}$ function defined on a neighborhood of the origin such that $f(0, x)=0$. Then $f(t, x)=\sum_{j=1}^{d} t_{j} g^{j}(t, x)$ for some $C^{\infty}$ functions $g^{1}, \ldots, g^{d}$ defined on a smaller neighborhood of the origin.

Proof of Theorem 2.2. (i) $\Rightarrow$ (ii): Choose independent 1 -forms $\omega^{1}, \ldots, \omega^{n}$ so that

$$
d \rho_{1}, \ldots, d \rho_{m-n}, \omega^{1}, \ldots, \omega^{n}
$$

$\operatorname{span} T^{*} M$. Then

$$
i^{*}\left(\omega^{1} \wedge \cdots \wedge \omega^{n}\right) \neq 0 .
$$


Set

$$
\theta^{\alpha}=\sum_{j=1}^{m-n} a^{\alpha j} d \rho_{j}+\sum_{j=1}^{n} b_{j}^{\alpha} \omega^{j} .
$$

Since $i^{*} \theta^{\alpha}=0$ and $i^{*}\left(d \rho_{j}\right)=0$, pulling back (2.2) by $i$ we have

$$
0=\sum_{j=1}^{n} b_{j}^{\alpha}\left(i^{*} \omega^{j}\right)
$$

Therefore, for each $\alpha, j$, we have $b_{j}^{\alpha}=0$ on $N$. Then by Lemma 2.3 we have

$$
b_{j}^{\alpha}=\sum_{k=1}^{m-n} h_{j}^{\alpha k} \rho_{k}
$$

for some smooth function $h_{j}^{\alpha k}$. Substituting (2.3) for $b_{j}^{\alpha}$ in (2.2) we have

$$
\theta^{\alpha}=\sum_{j=1}^{m-n} a^{\alpha j} d \rho_{j}+\sum_{j=1}^{n} \sum_{k=1}^{m-n} \rho_{k} h_{j}^{\alpha k} \omega^{j}
$$

(ii) $\Rightarrow$ i): Suppose that

$$
\theta^{\alpha}=\sum_{j=1}^{m-n} \rho_{j} \psi^{\alpha j}+\sum_{j=1}^{m-n} h^{\alpha j} d \rho_{j}
$$

for some 1-forms $\psi^{\alpha j}$ and smooth functions $h^{\alpha j}$. Apply any tangent vector $(x, V) \in T N$ to $(2.5)$. Since $\rho_{j}(x)=0$ and $d \rho_{j}(V)=0$, we have $\theta^{\alpha}(V)=0$, which implies that $i^{*} \theta^{\alpha}=0$.

Now we study by using Theorem 2.2, the existence of integral manifold $i: N^{n} \hookrightarrow M^{m}, 2 \leq n \leq p$, of the Pfaffian system

$$
\theta^{\alpha}=0, \quad \alpha=1, \ldots, s, \quad s+p=m .
$$

Suppose that $N$ is an integral manifold of (2.6). Then $i^{*} \theta^{\alpha}=0$ implies that $d\left(i^{*} \theta^{\alpha}\right)=i^{*}\left(d \theta^{\alpha}\right)=0$. Let $\omega^{1}, \ldots, \omega^{p}$ be the complementary set of 1 -forms. We set as usual

$$
d \theta^{\alpha}=\sum_{i, j=1}^{p} T_{i j}^{\alpha} \omega^{i} \wedge \omega^{j}, \quad \bmod \theta, \quad \alpha=1, \ldots, s,
$$

where $T_{j i}^{\alpha}=-T_{i j}^{\alpha}$. Consider $\left(\begin{array}{l}p \\ 2\end{array}\right):=p(p-1) / 2$ linearly independent differential 2 -forms $\omega^{i} \wedge \omega^{j}$ arranged in lexico-graphical order. Let

$$
\mathcal{T}=\left(T_{i j}^{\alpha}\right)
$$

be the matrix of size $s \times\left(\begin{array}{l}p \\ 2\end{array}\right)$. We shall call $\mathcal{T}$ torsion of the Pfaffian system (2.6). 
Proposition 2.4. Let $M$ be a smooth manifold of dimension $m$ and let $\theta^{1}, \ldots$, $\theta^{s}, \omega^{1}, \ldots, \omega^{p}$ be a system of smooth real 1-forms as in (2.6)-(2.7). Suppose that $N$ is an integral manifold of (2.6) of dimension $n, 2 \leq n \leq p$. Then there exists $\left(\begin{array}{l}p \\ 2\end{array}\right) \times\left(\begin{array}{l}n \\ 2\end{array}\right)$ matrix valued smooth function $A$ of rank $\left(\begin{array}{l}n \\ 2\end{array}\right)$ defined on $N$ such that

$$
\mathcal{T} A=0 .
$$

In particular, if $N^{p}$ is an integral manifold of maximal dimension, then $\mathcal{T}=0$ on $N^{p}$.

Proof. After re-ordering if necessary, we may assume that $\left.\omega^{1} \wedge \cdots \wedge \omega^{n}\right|_{N} \neq 0$. Set

$$
\left.\omega^{\lambda}\right|_{N}=\left.\sum_{i=1}^{n} a_{i}^{\lambda} \omega^{i}\right|_{N}, \quad \lambda=n+1, \ldots, p .
$$

Then the restriction to $N$ of (2.7) becomes

$$
\begin{aligned}
0 & =\sum_{\substack{i<j \\
i, j=1, \ldots, n}} \tau_{i j}^{\alpha} \omega^{i} \wedge \omega^{j}, \text { where } \\
\tau_{i j}^{\alpha} & =T_{i j}^{\alpha}+\sum_{\mu=n+1}^{p} T_{i \mu}^{\alpha} a_{j}^{\mu}-\sum_{\lambda=n+1}^{p} T_{j \lambda}^{\alpha} a_{i}^{\lambda}+\sum_{\substack{\lambda<\mu \\
\lambda, \mu=n+1, \ldots, p}} T_{\lambda \mu}^{\alpha}\left(a_{i}^{\lambda} a_{j}^{\mu}-a_{j}^{\lambda} a_{i}^{\mu}\right),
\end{aligned}
$$

$\alpha=1, \ldots, s$. Since $\omega^{i} \wedge \omega^{j}, i<j$, are independent on $N,(2.11)$ implies

$$
T_{i j}^{\alpha}+\sum_{\mu=n+1}^{p} T_{i \mu}^{\alpha} a_{j}^{\mu}-\sum_{\lambda=n+1}^{p} T_{j \lambda}^{\alpha} a_{i}^{\lambda}+\sum_{\substack{\lambda<\mu \\ \lambda, \mu=n+1, \ldots, p}} T_{\lambda \mu}^{\alpha}\left(a_{i}^{\lambda} a_{j}^{\mu}-a_{j}^{\lambda} a_{i}^{\mu}\right)=0
$$

for each $\alpha=1, \ldots, s$ and each pair $(i j)$ with $i<j, i, j=1, \ldots, n$. In matrices we write $(2.12)$ as

$$
\mathcal{T} A=0,
$$

where $A$ is a matrix of size $\left(\begin{array}{l}p \\ 2\end{array}\right) \times\left(\begin{array}{l}n \\ 2\end{array}\right)$ given as follows: for a pair $I=(i j)$ with $i<j, i, j=1, \ldots, n, I$-th column of $A$ is

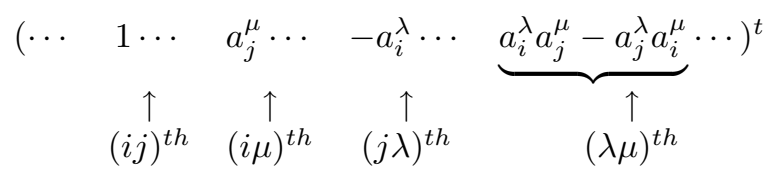

for $n<\lambda<\mu$, and dots are all zeros. Observe that the first $\left(\begin{array}{l}n \\ 2\end{array}\right)$ rows or $A$ is the identity matrix, therefore $A$ is of maximal rank. In particular, if $n=p$ then $A$ is the identity matrix of size $\left(\begin{array}{l}p \\ 2\end{array}\right)$, therefore, $\mathcal{T}$ is identically zero on an integral manifold of maximal dimension $p$.

Observe that $(2.13)$ is a system of $\left(\begin{array}{l}n \\ 2\end{array}\right)$ independent linear equations on the $\left(\begin{array}{l}p \\ 2\end{array}\right)$ columns of $\mathcal{T}$. Hence we have: 
Theorem 2.5. If $N$ is an integral manifold of (2.6) of dimension $n, 2 \leq n \leq p$, then the number of linearly independent columns of $\mathcal{T}$ is at most $\left(\begin{array}{l}p \\ 2\end{array}\right)-\left(\begin{array}{l}n \\ 2\end{array}\right)$.

Definition 2.6. Given a set of smooth functions $T_{\alpha}, \alpha=1, \ldots, k$ on $M$ a smooth real-valued function $\rho$ is said to be a common factor of $T_{\alpha}$ 's if $T_{\alpha}=\rho \phi_{\alpha}$, for some smooth function $\phi_{\alpha}$ for each $\alpha=1, \ldots, k$.

Theorem 2.7. Let $\theta^{1}, \ldots, \theta^{s}, \omega^{1}, \ldots, \omega^{p}$ be real 1 -forms of $M^{m}, s+p=m$, as in (2.6)-(2.7). Let $n, 2 \leq n \leq p$, be an integer. Then there exists an integral manifold $N$ of (2.6) of dimension $n$ if and only if there exists a non-degenerate set of real-valued functions $\rho=\left(\rho_{1}, \ldots, \rho_{m-n}\right)$ having the following properties: on the common zero locus of $\rho$ the first $\left(\begin{array}{l}n \\ 2\end{array}\right)$ columns (after rearrangement) $\mathcal{T}_{1}, \ldots, \mathcal{T}_{\left(\begin{array}{c}n \\ 2\end{array}\right)}$ belong to the linear span of $\mathcal{T}_{\lambda}, \lambda=\left(\begin{array}{l}n \\ 2\end{array}\right)+1, \ldots,\left(\begin{array}{l}p \\ 2\end{array}\right)$, where $\mathcal{T}_{\lambda}$ is the $\lambda$-th column of $\mathcal{T}$, and

$$
\theta^{\alpha}=0, \bmod (\rho, d \rho) .
$$

Then $N$; the common zero locus of $\rho$ is an integral manifold of dimension $n$.

Generalization of the Frobenius theorem for the existence of a single integral manifold of maximal dimension $p$ is found in [16]. Our results [9], [10] and [11] on the generalization of the Frobenius theorem are obtained independently and comprises more general cases: existence of $s^{\prime}$-parameter $\left(s^{\prime} \leq s\right)$ family of integral manifolds of dimension $p$ and existence of integral manifolds of dimension $p^{\prime}\left(p^{\prime} \leq p\right)$.

\section{$\S 3$. Existence of complex submanifolds in terms of derivatives of the Levi-form}

Let $M$ be a smooth $\left(C^{\infty}\right)$ real hypersurface in $\mathbb{C}^{n+1}$, with coordinates $(z, w)$, where $z=\left(z_{1}, \ldots, z_{n}\right)$, defined on a neighborhood $U$ of our reference point $P$. Let $M$ be defined by $r(z, \bar{z}, w, \bar{w})=0$, where $r$ is a $C^{\infty}$ non-degenerate realvalued function defined on an open subset $U$ of $M$. We assume $r_{w} \neq 0$. In this section we discuss conditions for $M$ to admit complex submanifolds through $P$. Our strategy is to find a set of $C^{\infty}$ non-degenerate real-valued functions $\rho=\left(\rho_{1}, \ldots, \rho_{d}\right)$ that defines a submanifold $N \subset M$ as in Theorem 2.2, where $\rho$ 's are obtained from the rank condition of the torsion tensor as in Theorem 2.7. In addition, we require the tangent bundle of $N$ to be invariant under the complex structure tensor $J$ of $\mathbb{C}^{n+1}$. For the common zero locus $N$ of $\rho$ the following conditions are equivalent as we shall see in Theorem 3.5:

1) $N$ is a complex manifold.

2) $J(T N) \subset T N$.

3) $\theta \equiv 0$ and $\theta^{\nu} \equiv 0, \nu=1, \ldots, d$, modulo $(r, \rho, d r, d \rho)$, where $\theta^{\nu}:=$ $\sqrt{-1} \partial \rho_{\nu}$.

In this section we adopt from [1] and [3] the standard definitions and notations of CR geometry. We first present a necessary and sufficient condition for 
a complex hypersurface to exist through $P$ and then extend our argument to the cases of complex submanifolds of higher codimensions.

Let

$$
\theta=\sqrt{-1} \partial r
$$

Since $d r=\partial r+\bar{\partial} r=0$ on $M$ we have $\bar{\theta}=-\sqrt{-1} \bar{\partial} r=\sqrt{-1} \partial r=\theta$, therefore, $\theta$ is a real 1 -form on $M$. Then

$$
H(M):=\{v \in T(M): \theta(v)=0\}
$$

is the bundle of maximal complex subspaces of $T(M)$. A real submanifold $N \subset M$ of dimension $2 n$ is a complex submanifold if and only if $N$ is an integral manifold of $H(M)$ and $T N$ is $J$-invariant. For the sake of computational convenience we use the ambient coordinates $(z, w)$ of $\mathbb{C}^{n+1}$. Thus our problem is to find a $J$-invariant integral manifold of dimension $2 n$ of the exterior differential system

$$
(r, \theta)
$$

If $N$ is an integral manifold of (3.2), then $\left.r\right|_{N}=0$ and $\left.\theta\right|_{N}=0$, therefore, $\left.d r\right|_{N}=0$ and $\left.d \theta\right|_{N}=0$. Since $\theta=\bar{\theta}, \bmod (d r)$, and

$$
\frac{1}{\sqrt{-1}} \theta=\sum_{i=1}^{n} r_{i} d z_{i}+r_{w} d w
$$

we have

$$
\begin{array}{ll}
d w=-\frac{1}{r_{w}} \sum r_{j} d z_{j}, & \bmod (d r, \theta) \\
d \bar{w}=-\frac{1}{r_{\bar{w}}} \sum r_{\bar{j}} d \bar{z}_{j}, & \bmod (d r, \theta) .
\end{array}
$$

Since

$$
\begin{aligned}
\frac{1}{\sqrt{-1}} d \theta & =\bar{\partial} \partial r \\
& =\sum_{i, j=1}^{n}\left\{r_{i \bar{j}} d \bar{z}_{j} \wedge d z_{i}+r_{i \bar{w}} d \bar{w} \wedge d z_{i}+r_{w \bar{j}} d \bar{z}_{j} \wedge d w+r_{w \bar{w}} d \bar{w} \wedge d w\right\}
\end{aligned}
$$

by substituting (3.3) for $d w$ and for $d \bar{w}$ we have

$$
\frac{1}{\sqrt{-1}} d \theta \equiv \sum_{i, j=1}^{n} T_{i \bar{j}} d \bar{z}_{j} \wedge d z_{i}, \quad \bmod (d r, \theta),
$$

where

$$
T_{i \bar{j}}=r_{i \bar{j}}-r_{i \bar{w}} \frac{r_{\bar{j}}}{r_{\bar{w}}}-r_{w \bar{j}} \frac{r_{i}}{r_{w}}+r_{w \bar{w}} \frac{r_{\bar{j}}}{r_{\bar{w}}} \frac{r_{i}}{r_{w}} .
$$

We shall call the Hermitian matrix $\left(T_{i \bar{j}}\right)$ the Levi matrix. This is the matrix of the coefficients of the Levi form of $M$. If $M$ is Levi flat, that is, if

$$
T_{i \bar{j}} \equiv 0, \bmod (r), \quad \forall i, j=1, \ldots, n
$$


then by the Frobenius theorem $M$ is foliated by complex hypersurfaces. The functions $T_{i \bar{j}}$, mod $(r)$, are the obstruction to the existence of integral manifolds, which is generally called torsion for the exterior differential system (3.2).

Definition 3.1. A real valued function $\rho$ defined on $U$ is a factor of the Leviform $\left(T_{i \bar{j}}\right)$ if $T_{i \bar{j}} \equiv 0, \bmod (r, \rho)$ for each $i, j$.

Our main observation is that if a complex hypersurface exits it is given as the zero locus of a nondegenerate factor $\rho$ of the Levi-form. A necessary and sufficient condition for the existence of a complex hypersurface is that $\theta(v)=0$ for all vectors $v \in T_{x} \mathbb{C}^{n+1}$ with $r(x)=\rho(x)=0, d r(v)=0$ and $d \rho(v)=0$, which is a condition on the derivatives of $r$ up to third order. We have:

Theorem 3.2. Let $M$ be a real hypersurface in $\mathbb{C}^{n+1}, n \geq 1$, given as a zero locus of a smooth real-valued function $r$ with $r_{w} \neq 0$ defined on a small neighborhood $U \subset \mathbb{C}^{n+1}$ of a point $P \in M$. Let $\theta$ and $T_{i \bar{j}}$ be the same as defined by (3.1) and (3.4). Then there exists a complex hypersurface $N$ in $M$ through $P$ if and only if there is a factor $\rho$ of the Levi-form such that

i) $\rho(P)=0, \quad d r \wedge d \rho \neq 0$.

ii) $\theta \equiv 0, \quad \bmod (r, \rho, d r, d \rho)$.

Proof. Suppose that $N$ is a complex hypersurface through $P$. Then

$$
\begin{aligned}
0 & =\left.\frac{1}{\sqrt{-1}} d \theta\right|_{N} \\
& =\sum_{i, j=1}^{n}\left(\left.T_{i \bar{j}}\right|_{N}\right) d \bar{z}_{j} \wedge d z_{i} .
\end{aligned}
$$

Since $d \bar{z}_{j} \wedge d z_{i}$ are independent on $N$, we have $\left.T_{i \bar{j}}\right|_{N}=0$ for each $i, j=1, \ldots, n$. Now choose any smooth real-valued function $\rho$ on $U$ such that $N$ is the common zero set of $r$ and $\rho$ and such that $d \rho \wedge d r \neq 0$ on $N$. We take a local coordinate system $\left(r, \rho, x_{1}, \ldots, x_{2 n}\right)$ of $\mathbb{C}^{n+1}$. Then $T_{i \bar{j}} \equiv 0, \bmod (r, \rho)$. Now ii) follows from observing that the following are equivalent:

a) $T_{x} N=H_{x}(M), \quad \forall x \in N$.

b) For $x \in N$ and for $v \in T_{x} N$ we have $\theta(v)=0$.

c) For $v \in T_{x}\left(\mathbb{C}^{n+1}\right)$ with $r(x)=\rho(x)=0, d r(v)=0, d \rho(v)=0$ we have $\theta(v)=0$.

d) $\theta \equiv 0, \bmod (r, \rho, d r, d \rho)$.

Conversely, suppose that $\rho$ is a factor of the Levi-form with the properties i) and ii). Let $N$ be the common zero set of $r$ and $\rho$. Then the property i) implies that $N$, near $P$, is a smooth (2n)-dimensional submanifold of $M$ containing $P$ and ii) implies that any tangent vector to $N$ belongs to $H(M)$, hence, $N$ is a complex hypersurface.

Example 3.3. Quadric real hypersurfaces in $\mathbb{C}^{2}$ : Let $Q$ be the zero set of

$$
r=w+\bar{w}+a z \bar{z}+\lambda z \bar{w}+\bar{\lambda} w \bar{z}+b w \bar{w},
$$


where $a, b \in \mathbb{R}$, and $\lambda \in \mathbb{C}$ are constants.

We shall show that if $Q$ contains a complex hypersurface through the origin, then $Q$ is Levi flat. We have

$$
\theta=\sqrt{-1}\{(a \bar{z}+\lambda \bar{w}) d z+(1+\bar{\lambda} \bar{z}+b \bar{w}) d w\}
$$

and

$$
\begin{aligned}
\frac{1}{\sqrt{-1}} d \theta & =\bar{\partial} \partial r \\
& \equiv T d \bar{z} \wedge d z, \quad \bmod (\theta, d r)
\end{aligned}
$$

where

$$
T=a-\lambda \frac{\bar{a} z+\bar{\lambda} w}{1+\lambda z+\bar{b} w}-\bar{\lambda} \frac{a \bar{z}+\lambda \bar{w}}{1+\bar{\lambda} \bar{z}+b \bar{w}}+b \frac{\bar{a} z+\bar{\lambda} w}{1+\lambda z+\bar{b} w} \frac{a \bar{z}+\lambda \bar{w}}{1+\bar{\lambda} \bar{z}+b \bar{w}} .
$$

Let $\mathcal{T}$ be $T$ multiplied by the common denominator:

$$
\begin{aligned}
\mathcal{T}= & a+(a b-\lambda \bar{\lambda}) w+(a b-\lambda \bar{\lambda}) \bar{w}+\left(-\lambda \bar{\lambda} a+b a^{2}\right) z \bar{z} \\
& +\left(-\lambda \bar{\lambda}^{2}+b \bar{\lambda} a\right) \bar{z} w+\left(-\bar{\lambda} \lambda^{2}+b a \lambda\right) z \bar{w}+\left(a b^{2}-\lambda \bar{\lambda} b\right) w \bar{w} .
\end{aligned}
$$

Therefore, in order for the origin to be a zero of $\mathcal{T}$ the coefficient a must be zero and in that case $Q$ contains the complex line $w=0$. We have

$$
r=w+\bar{w}+\lambda z \bar{w}+\bar{\lambda} w \bar{z}+b w \bar{w}
$$

and

$$
\mathcal{T}=-\lambda \bar{\lambda}(w+\bar{w})-\lambda \bar{\lambda}^{2} \bar{z} w-\bar{\lambda} \lambda^{2} z \bar{w}-\lambda \bar{\lambda} b w \bar{w}
$$

Observe that

$$
\mathcal{T}=-\lambda \bar{\lambda} r \equiv 0, \quad \bmod (r)
$$

therefore $Q$ is Levi flat.

Example 3.4. Cubic real hypersurfaces in $\mathbb{C}^{2}=\{(z, w)\}$ : Let $z=x+i y$ and $w=u+i v$. Consider the zero set $M$ of

$$
\begin{aligned}
r & =2 u(1+2 y)+8 v x^{2} \\
& =(w+\bar{w})\left(1+\frac{z-\bar{z}}{i}\right)+\frac{w-\bar{w}}{i}(z+\bar{z})^{2} .
\end{aligned}
$$

We shall show that $M$ is not Levi flat and a complex line $w=0$ is contained in $M$. We have $d r=16 x v d x+4 u d y+2(1+2 y) d u+8 x^{2} d v$ and

$$
\begin{aligned}
\theta & =i \partial r \\
& =[w+\bar{w}+2(w-\bar{w})(z+\bar{z})] d z+\left[i+z-\bar{z}+(z+\bar{z})^{2}\right] d w,
\end{aligned}
$$

therefore,

$$
\begin{aligned}
& d w=-\frac{w+\bar{w}+2(w-\bar{w})(z+\bar{z})}{i+z-\bar{z}+(z+\bar{z})^{2}} d z, \quad \bmod \theta \\
& d \bar{w}=-\frac{w+\bar{w}-2(w-\bar{w})(z+\bar{z})}{-i+\bar{z}-z+(z+\bar{z})^{2}} d \bar{z}, \quad \bmod \theta
\end{aligned}
$$


Then

$$
\begin{aligned}
d \theta= & i \bar{\partial} \partial r \\
= & {[2(w-\bar{w}) d \bar{z}+(1-2(z+\bar{z})) d \bar{w}] \wedge d z+[-1+2(z+\bar{z})] d \bar{z} \wedge d w } \\
& (\text { substituting the above for } d w \text { and } d \bar{w}) \\
= & T d \bar{z} \wedge d z
\end{aligned}
$$

where

$$
\begin{array}{r}
T=2(w-\bar{w})-(1-2(z+\bar{z})) \frac{w+\bar{w}-2(w-\bar{w})(z+\bar{z})}{-i+\bar{z}-z+(z+\bar{z})^{2}} \\
-(-1+2(z+\bar{z})) \frac{w+\bar{w}+2(w-\bar{w})(z+\bar{z})}{i+z-\bar{z}+(z+\bar{z})^{2}} \\
=4 i v-(1-4 x) \frac{2 u-4 v i \cdot 2 x}{-i-2 y i+4 x^{2}}-(-1+4 x) \frac{2 u+4 v i \cdot 2 x}{i+2 y i+4 x^{2}} .
\end{array}
$$

To see that $M$ is not Levi flat consider a curve $\sigma(x)=\left(x, 0,-4 x^{3}, x\right)$, which lies on $M$ and passes through the origin. Observe that $T(\sigma(x))$, after multiplying by the product of the denominators, is a polynomial in $x$ of degree 6 without constant term, therefore, does not vanish identically. We also have

$$
d T=\zeta_{1}(x, y, u, v) d x+\zeta_{2}(x, y, u, v) d y+a d u+(4 i+\zeta) d v,
$$

where $\zeta_{j}(x, y, 0,0)=0$ for $j=1,2$ and $\zeta(0)=0$. The submanifold $r=T=0$ is the complex line $w=0$, along which we have

$$
\begin{aligned}
& d T=a d u+(4 i+\zeta) d v, \\
& d r=2(1+2 y) d u+8 x^{2} d v,
\end{aligned}
$$

and

Thus we have

$$
\begin{aligned}
\theta & =\left(i+z-\bar{z}+(z+\bar{z})^{2}\right) d w \\
& =\left(i+2 i y+4 x^{2}\right)(d u+i d v) .
\end{aligned}
$$

$$
\theta \equiv 0, \quad \bmod (r, T, d r, d T) .
$$

Now we discuss the cases of complex submanifolds of higher codimensions. First of all, we prove the following:

Theorem 3.5. Suppose that $N$ is a real submanifold of real dimension $2 k$ in $\mathbb{C}^{m}, 1 \leq k \leq m-1$, and that $N$ is locally given as the common zero set of non-degenerate real-valued functions $\rho:=\left(\rho_{1}, \ldots, \rho_{2 d}\right), d+k=m$. Then the following are equivalent:

a) $N$ is a complex submanifold of $\mathbb{C}^{m}$.

b) $N$ is $J$-invariant, that is, $J(T N) \subset T N$.

c) $\partial \rho_{j} \equiv 0, \bmod (\rho, d \rho)$ for each $j=1, \ldots, 2 d$, or equivalently,

$$
\theta^{j} \equiv 0, \quad \bmod (\rho, d \rho), \quad \text { where } \theta^{j}:=\sqrt{-1} \partial \rho_{j} .
$$


Proof. a) $\Longleftrightarrow$ b): We may assume $N$ is the graph

$$
z_{k+\lambda}=f_{\lambda}\left(z_{1}, \ldots, z_{k}\right), \quad \lambda=1, \ldots, d,
$$

where $f_{\lambda}$ are complex-valued functions. Then ii) is equivalent to that each $f_{\lambda}$ satisfies the Cauchy-Riemann equations, so that $f_{\lambda}$ are holomorphic.

b) $\Longrightarrow$ c): Recall that $d \rho_{j}=\partial \rho_{j}+\bar{\partial} \rho_{j}$ for $j=1, \ldots, 2 d$. Recall also that for any tangent vector $v$ of $\mathbb{C}^{m} v-\sqrt{-1} J v$ is a complex vector of type $(1,0)$ and $v+\sqrt{-1} J v$ is a complex vector of type $(0,1)$, so that

$$
\partial \rho_{j}(v+\sqrt{-1} J v)=0
$$

and

$$
\partial \rho_{j}(v-\sqrt{-1} J v)=d \rho_{j}(v-\sqrt{-1} J v) .
$$

Now suppose that $N$ is $J$-invariant. Then for any tangent vector $v$ to $N$ at $x \in N$ we have $J v \in T_{x} N$, so that $d \rho_{j}(J v)=0$. Therefore, by (3.7) and (3.6) we have for each $j=1, \ldots, 2 d$

$$
\begin{aligned}
& \partial \rho_{j}(v-\sqrt{-1} J v)=d \rho_{j}(v-\sqrt{-1} J v)=0, \\
& \partial \rho_{j}(v+\sqrt{-1} J v)=0
\end{aligned}
$$

(3.8) implies that

$$
\partial \rho_{j} \in\left(T_{\mathbb{C}} N\right)^{\perp} \subset(\rho, d \rho)
$$

where $(\rho, d \rho)$ denotes the algebraic ideal generated by $\rho_{1}, \ldots, \rho_{d}, d \rho_{1}, \ldots, d \rho_{2 d}$.

c) $\Longrightarrow$ b): Suppose that $\partial \rho_{j} \in(\rho, d \rho)$ for each $j=1, \ldots, d$. Then

$$
\partial \rho_{j}=\sum_{\alpha=1}^{2 d}\left(\rho_{\alpha} \phi^{\alpha}+a^{\alpha} d \rho_{\alpha}\right)
$$

for some smooth 1-forms $\phi^{\alpha}$ and functions $a^{\alpha}$. For any $v \in T_{x} N$ applying (3.9) to the complex vectors $(v-\sqrt{-1} J v)$ and $(v+\sqrt{-1} J v)$, respectively, we have

$$
\begin{aligned}
\partial \rho_{j}(v-\sqrt{-1} J v) & =\sum_{\alpha=1}^{2 d} a^{\alpha}(x) d \rho_{\alpha}(v-\sqrt{-1} J v) \\
& =\sum_{\alpha=1}^{2 d} a^{\alpha}(x)(-\sqrt{-1}) d \rho_{\alpha}(J v)
\end{aligned}
$$

and

$$
\begin{aligned}
0=\partial \rho_{j}(v+\sqrt{-1} J v) & =\sum_{\alpha=1}^{2 d} a^{\alpha}(x) d \rho_{\alpha}(v+\sqrt{-1} J v) \\
& =\sum_{\alpha=1}^{2 d} a^{\alpha}(x) \sqrt{-1} d \rho_{\alpha}(J v) .
\end{aligned}
$$

But the LHS of (3.10) is equal to

$$
d \rho_{j}(v-\sqrt{-1} J v)=-\sqrt{-1} d \rho_{j}(J v) .
$$


Hence, (3.10) becomes

$$
\sqrt{-1} d \rho_{j}(J v)=\sum_{\alpha=1}^{2 d} a^{\alpha}(x) \sqrt{-1} d \rho_{\alpha}(J v) .
$$

Subtract (3.11) from (3.12), to obtain

$$
d \rho_{j}(J v)=0, \quad j=1, \ldots, 2 d,
$$

which implies $J v \in T_{x} N$.

Now we find defining functions $\rho$ of a complex submanifold $N$ of a real hypersurface $M$ in $\mathbb{C}^{n+1}$. We shall modify the method of Theorem 2.7 to be suitable to the ambient complex structure. At the reference point $P$ we assume

$$
\operatorname{rank}\left[T_{i \bar{j}}\right]=n-k, \quad 1 \leq k \leq n .
$$

Suppose that $N^{2 k}$ is a complex submanifold of complex dimension $k$, through $P$. Then the rank condition implies that there is no other complex manifold through $P$ that is transversal to $N$. The Levi-form restricted to $N$ is zero, that is,

$$
d \theta(L, \bar{L}):=\sqrt{-1} \sum_{i, j=1}^{n} T_{i j} a_{i} \bar{a}_{j}=0
$$

if and only if the complex vector $L=\left(a_{1}, \ldots, a_{n}, b\right) \in \mathbb{C}^{n+1}$ is tangent to $N$. Therefore, at $P$ the null space of the Levi form is of complex dimension $k$.

Let $\tau_{1}, \ldots, \tau_{m}$ be the determinant of the square submatrices of $\left[T_{i \bar{j}}\right]$ of size $n-k+1$. Then each $\tau_{j}$ is a polynomial in $T_{i \bar{j}}$ of degree $n-k+1$. Then $(3.13)$ is equivalent to

$$
\left.\tau_{j}\right|_{N}=0
$$

Thus a complex submanifold $N$ is contained in the common zero locus of $\tau_{j}, j=1, \ldots, m$. If $N$ is defined as a common zero locus of real-valued functions $r, \rho_{1}, \ldots, \rho_{d}$ with $d r \wedge d \rho_{1} \wedge \cdots \wedge d \rho_{d} \neq 0$, where $d=2 n+1-2 k$, then each $\tau_{j}$ must be zero on the common zero locus of $r, \rho_{1}, \ldots, \rho_{d}$. This implies $\tau_{j} \equiv 0, \bmod \left(r, \rho_{1}, \ldots, \rho_{d}\right)$. For each $\rho_{\nu}, \nu=1, \ldots, d$, let $\theta^{\nu}=\sqrt{-1} \partial \rho_{\nu}$. Then by Theorem 3.5 the common zero set of $r, \rho_{1}, \ldots, \rho_{d}$ is a complex manifold if and only if $\theta \equiv 0$, and $\theta^{\nu} \equiv 0$, modulo $\left(r, \rho_{1}, \ldots, \rho_{d}, d r, d \rho_{1}, \ldots, d \rho_{d}\right)$. Thus we have the following:

Theorem 3.6. Let $M$ be a real hypersurface in $\mathbb{C}^{n+1}, n \geq 1$, given as the zero locus of a smooth real-valued function $r$ with $d r \neq 0$ defined on a small neighborhood $U \subset \mathbb{C}^{n+1}$ of a point $P \in M$. Let $\theta$ and $T_{i \bar{j}}$ be the same as defined by (3.1) and (3.4). Suppose that $\left[T_{i \bar{j}}\right]$ has rank $n-k$ at $P$. Then there exists a complex submanifold $N$ of complex dimension $k$ through $P$ if and only if there is a set of smooth real-valued functions $\rho_{1}, \ldots, \rho_{d}$, where $d=2 n+1-2 k$, defined on $U$ such that

i) For each $\nu=1, \ldots, d, \rho_{\nu}(P)=0$, and $d r \wedge d \rho_{1} \wedge \cdots \wedge d \rho_{d} \neq 0$, 
ii) Each $\tau_{j}, j=1, \ldots, m$, of (3.14) is zero modulo $\left(r, \rho_{1}, \ldots, \rho_{d}\right)$,

iii) $\theta \equiv 0$ and $\theta^{\nu} \equiv 0$ for each $\nu$, modulo $\left(r, \rho_{1}, \ldots, \rho_{d}, d r, d \rho_{1}, \ldots, d \rho_{d}\right)$.

Example 3.7. Complex curve through the origin in $M^{5} \subset \mathbb{C}^{3}=\left\{\left(z_{1}, z_{2}, w\right)\right\}$ : Let $M$ be the zero locus of

$$
r=w+\bar{w}+a z_{1} \bar{z}_{1}+\lambda\left(z_{1}\right)^{2} \bar{z}_{2}+\bar{\lambda} z_{2}\left(\bar{z}_{1}\right)^{2},
$$

where $a$ is a real constant and $\lambda$ is a nonzero complex constant.

In this example

$$
T:=\left[T_{i \bar{j}}\right]=\left[\begin{array}{cc}
a & 2 \lambda z_{1} \\
2 \bar{\lambda} \bar{z}_{1} & 0
\end{array}\right]
$$

so that $\operatorname{det} T=-4 \lambda \bar{\lambda} z_{1} \bar{z}_{1}, T$ has rank 2 if $z_{1} \neq 0$.

Case $a \neq 0$ : If $z_{1}=0$ the Levi matrix $T$ has rank 1 , in particular, $T$ has rank 1 at the origin. Thus we apply Theorem 3.6 with $n=2, k=1$ and $d=2 n+1-2 k=3$. We take $\rho_{1}=\Im w, \rho_{2}=\Re z_{1}$, and $\rho_{3}=\Im z_{1}$. Let $N$ be the set of common zeros of $\left(r, \rho_{\nu}, \nu=1,2,3\right)$, which is a complex line $(0, \zeta, 0)$. Then modulo $\left(r, \rho_{1}, \rho_{2}, \rho_{3}\right)$ we have

$$
\begin{array}{cc}
d r=d w+d \bar{w}, & \theta=\sqrt{-1} d w \\
d \rho_{1}=\frac{1}{2 \sqrt{-1}}(d w-d \bar{w}), & \theta^{1}=\frac{1}{2} d w \\
d \rho_{2}=\frac{1}{2}\left(d z_{1}+d \bar{z}_{1}\right), & \theta^{2}=\frac{\sqrt{-1}}{2} d z_{1} \\
d \rho_{3}=\frac{1}{2 \sqrt{-1}}\left(d z_{1}-d \bar{z}_{1}\right), & \theta^{3}=\frac{1}{2}\left(d z_{1}\right) .
\end{array}
$$

We see that $\theta \equiv 0$, and $\theta^{\nu} \equiv 0$ for $\nu=1,2,3, \bmod \left(r, \rho_{1}, \rho_{2}, \rho_{3}, d r, d \rho_{1}, d \rho_{2}, d \rho_{3}\right)$. Case $a=0$ : If $z_{1}=0$, then the Levi matrix $T$ has rank 0 , therefore, Theorem 3.6 is not applicable. $T$ being of rank 0 is a necessary condition for there to exist a complex 2-manifold. However, it does not imply the existence of complex 2-manifold. Through the origin there pass two complex curves transversally: $(0, \zeta, 0)$ and $(\zeta, 0,0), \zeta \in \mathbb{C}$.

\section{References}

[1] A. Boggess, CR Manifolds and the Tangential Cauchy-Riemann Complex, Studies in Advanced Mathematics. CRC Press, Boca Raton, FL, 1991.

[2] R. Bryant, S. S. Chern, R. Gardner, H. Goldschmidt, and P. Griffiths, Exterior Differential Systems, Springer-Verlag, New York, 1986

[3] S. S. Chern and J. K. Moser, Real hypersurfaces in complex manifolds, Acta Math. 133 (1974), 219-271.

[4] G. Darboux, Sur le problème de Pfaff (1), (2), Bull.Sci. Math. 6 (1882), 14-36, 49-68.

[5] K. Diederich and J. Fornaess, Pseudoconvex domains with real-analytic boundary, Ann. Math. (2) 107 (1978), no. 2, 371-384.

[6] N. G. Dieu, Real hypersurface containing complex varieties, preprint.

[7] G. Frobenius, Über das Pfaffsche probleme, J. Reine und Angew. Math. 82 (1877), 230-315.

[8] P. Griffiths and G. Jensen, Differential Systems and Isometric Embeddings, Annals of Mathematics Studies, 114. The William H. Roever Lectures in Geometry. Princeton University Press, Princeton, NJ, 1987. 
[9] C. K. Han, Solvability of overdetermined PDE systems that admit a complete prolongation and some local problems in CR geometry, J. Korean Math. Soc. 40 (2003), no. 4, 695-708.

[10] _ Pfaffian systems of Frobenius type and solvability of generic overdetermined $P D E$ systems, Symmetries and overdetermined systems of partial differential equations, 421-429, IMA Vol. Math. Appl., 144, Springer, New York, 2008.

[11] _ Generalization of the Frobenius theorem on involutivity, J. Korean Math. Soc. 46 (2009), no. 5, 1087-1103.

[12] M. Kuranishi, On E. Cartan's prolongation theorem of exterior differential systems, Amer. J. Math. 79 (1957), 1-47.

[13] H. Lewy, On the local character of the solutions of an atypical linear differential equation in three variables and a related theorem for regular functions of two complex variables, Ann. of Math. (2) 64 (1956), 514-522.

[14] J. M. Trépreau, The holomorphic extension of CR functions defined on a real $C^{2}$ hypersurface in $C^{n}$, Invent. Math. 83 (1986), no. 3, 583-592.

[15] A. Tumanov, Extension of CR-functions into a wedge, Mat. Sb. 181 (1990), no. 7, 951-964; translation in Math. USSR-Sb. 70 (1991), no. 2, 385-398.

[16] S. Wang, Exterior differential system, Lectures at Seoul Nat. Univ. based on R. Bryant's lectures at Duke Univ, 2005.

ChONG-KYU HAN

Department of Mathematics

Seoul National University

SEOUl 151-742, Korea

E-mail address: ckhan@snu.ac.kr

Giuseppe Tomassini

Scuola Normale Superiore

Piazza Dei Cavalieri, 7-1I-56126 Pisa, Italy

E-mail address: g.tomassini@sns.it 